\title{
Outcomes after Micronized Fat Adipose Transfer for Glenohumeral Joint Arthritis and Rotator Cuff Pathology: a Case Series of 18 Shoulders
}

\author{
D.M. Robinson ${ }^{1}$, C. Eng ${ }^{1,2}$, M. Mitchkash ${ }^{1}$, A.S. Tenforde ${ }^{1,3}$, J. Borg-Stein ${ }^{1,2}$ \\ 1 Spaulding Rehabilitation Hospital, Harvard Medical School, Department of Physical Medicine and Rehabilitation, \\ Charlestown, Massachusetts \\ 2 Spaulding Rehabilitation Hospital, Harvard Medical School, Department of Physical Medicine and Rehabilitation, \\ Spaulding Wellesley Outpatient Care Center, Newton Wellesley Hospital, Newton, Massachusetts \\ 3 Spaulding Rehabilitation Hospital, Harvard Medical School, Department of Physical Medicine and Rehabilitation, \\ Spaulding National Running Center, Cambridge, Massachusetts
}

\section{CORRESPONDING AUTHOR: \\ David Robinson \\ Spaulding Rehabilitation Hospital \\ Harvard Medical School \\ Department of Physical Medicine and \\ Rehabilitation \\ 300 1st Avenue \\ Charlestown, Massachusetts 02129 \\ E-mail: drobinson22@partners.org; \\ k09dr02@gmail.com \\ DOI: \\ 10.32098/mltj.03.2020.06}

LEVEL OF EVIDENCE: 4

\begin{abstract}
SUMMARY
Background. This study aimed to evaluate the safety and clinical outcomes of patients treated with micro-fragmented adipose tissue for shoulder pain secondary to glenohumeral osteoarthritis and rotator cuff pathology.

Methods. 16 patients (18 shoulders) who had failed previous conservative therapies and received a single injection of micro-fragmented adipose tissue for shoulder pathology. Outcomes including pain, disability, and safety were assessed at minimum of six months.

Results. Significant improvements in visual analog scale scores $(\mathrm{p}<0.001)$ and pain disability index scores $(\mathrm{p}=0.02)$ with no major adverse events were observed at six months.

Conclusions. Micro-fragmented adipose tissue may be helpful to improve pain and function in a subset of patients with chronic glenohumeral osteoarthritis and rotator cuff tears. No major complications were identified in our case series.
\end{abstract}

KEY WORDS

Medicinal signaling cells; MFAT; orthobiologics; regenerative medicine; shoulder

\section{INTRODUCTION}

Shoulder pain is a common debilitating condition with an estimated annual prevalence ranging from $4.7-46.7 \%$ (1). In 2008 , nearly 19 million adults ( $>8 \%$ of the US adult population) reported chronic shoulder pain (2). Rotator cuff disorders and glenohumeral osteoarthritis are two of the most common etiologies $(3,4)$. Patients frequently continue to suffer with pain despite treatment, with $40-50 \%$ having continuous or recurrent pain complaints at one year (5). Direct costs in 2000 for treating shoulder pain in the United States totaled $\$ 7$ billion (3).

Early in the clinical course of shoulder disorders typical treatments offered include activity modification, physical therapy, oral anti-inflammatories and corticosteroid injections (4). The long-term use of corticosteroid is becoming increasingly controversial, as there are concerns regarding tendon and chondral toxicity (6). Until recently, the next step in treatment was often surgical with rotator cuff repair or shoulder arthroplasty. Not all patients are candidates for repair secondary to a variety of patient and lesion factors, and post-operative recovery for arthroplasty has significant downtime $(7,8)$. Newer injection options such as platelet rich plasma and mesenchymal signaling cells (MSCs), the latter of which are typically obtained from autologous bone marrow or adipose tissue are now being increasingly used (9).

MSCs are cells with the perceived capability to differentiate into cells that regenerate tissue functionality following injury (10). Numerous mechanisms have been proposed to explain how MSCs may support tissue repair and relieve pain. Secretion of cytokines and growth factors through a 
paracrine mechanism likely plays a large role (11). This paracrine activity is thought to stimulate angiogenesis and have anti-inflammatory properties (12). Secondary to its abundant vasculature, adipose is an excellent medium for MSC harvest. The procurement procedure consists of a minimally invasive harvest with higher cell concentration per unit volume compared to bone marrow concentrate (13). Complex regulations limit utility of culture expansion techniques and enzymatic digestion to obtain stromal vascular fraction. Mechanical treatment with micro-fragmentation provides an effective means of obtaining minimally manipulated adipose tissue known as micro-fragmented adipose tissue (MFAT) for therapeutic use (14).

Limited literature is available on the outcomes of patients treated with MFAT for shoulder pain. Continued clinical use necessitates continued analysis of outcomes of the procedure. In the present study, we report the functional and pain outcomes of 16 patients (18 shoulders) treated with MFAT injections for glenohumeral osteoarthritis and rotator cuff tears.

\section{METHODS}

\section{Participants}

All patients with glenohumeral osteoarthritis or rotator cuff tears treated with adipose-derived stromal cells from November 2017 to May 2019 at a single outpatient sports medicine clinic were evaluated for inclusion. Diagnosis was obtained through history, examination, and imaging.

Inclusion criteria were: (1) primary diagnosis of glenohumeral osteoarthritis or rotator cuff tear, (2) pathology present on magnetic resonance imaging or plain radiography, (3) pain duration greater than 6 months, and (4) failed conservative management with any combination of physical therapy, corticosteroid injection, visco-supplementation, platelet rich plasma, or arthroscopy. Exclusion criteria were corticosteroid injection within 3 months of MFAT procedure, malignancy, active infection, or auto-immune arthritis.

Sixteen patients (18 shoulders) met criteria and were analyzed. Seventeen shoulders had pathology confirmed through MRI, and in one case severe glenohumeral osteoarthritis was confirmed with plain radiography.

\section{Procedure}

The Lipogems ${ }^{\circledR}$ procedural kit was used in all patients. This kit provides the necessary resources for the harvest, processing, and transfer of MFAT. Mild mechanical forces are employed to reduce adipose tissue cluster size and eliminate proinflammatory oil and blood residues (15). The resultant product is neither enzymatically treated nor culture expanded. All injections were performed by two experienced physiatrists using ultrasound guidance (CE, JBS). The following standardized procedural protocol was used:

Harvest site was determined in the standing position. The patient was subsequently placed supine or prone for flank and thigh harvest sites, respectively, and the skin overlying the harvest site was cleansed with chlorhexidine. After injection of local anesthetic, an 18-gauge needle tip was used to create a small incision. The site was infiltrated with tumescent anesthesia $(500 \mathrm{~mL}$ Normal Saline, $1 \mathrm{~mL}$ of 1:1000 Epinephrine, $50 \mathrm{~mL}$ of $1 \%$ Lidocaine $/ 25 \mathrm{~mL}$ of $2 \%$ Lidocaine, 5 $\mathrm{mL}$ of $8.4 \%$ Sodium Bicarbonate) using the 17 -gauge Lipogems ${ }^{\circledR}$ blunt anesthesia tip connected to a $60 \mathrm{~mL}$ syringe. The cannula was advanced laterally to medially to disperse the tumescent anesthesia. At least 20 minutes post completion of tumescent anesthesia, the lipoaspiration was started. Fat was harvested using a 13 -gauge lipoaspirate cannula connected to a $20 \mathrm{~mL}$ VacLok syringe. Care was taken to avoid any air being introduced to the syringe. The lipoaspirate syringes were placed in a sterile cup to decant and subsequently transferred to the Lipogems ${ }^{\circledR}$ device for processing. The final product was placed in $3 \mathrm{~mL}$ syringes for the treatment. As the lipoaspirate was being washing, excess tumescent was expressed from the harvest site and the puncture site was dressed in steri-strips and a sterile gauze, which was then covered by Tegaderm. Tape was placed along the harvest site to minimize swelling, bruising, and post-procedure pain. Ultrasound guidance with a curvilinear probe for the glenohumeral joint and linear probe for rotator cuff lesions was used for tissue transfer with an in-plane needle approach. Under sterile conditions, the final adipose aspirate was injected into the target site using a $18 \mathrm{~g} 3.5$-inch needle. Average MFAT injectate volume was $4.9 \pm 1.4 \mathrm{~mL}$ for glenohumeral arthritis and $1.4 \pm 0.6 \mathrm{~mL}$ for rotator cuff pathology. After completion of the procedure, the patient was monitored for 15-20 minutes and provided the post-procedure instructions, which were reviewed in-person.

\section{Assessments}

Approval was obtained from our institution's quality improvement advisory board; IRB approval was thereby waived by the institution. This study meets the ethical standards of the journal (16). Data was collected during routine clinic follow-up visits. The following outcomes were collected: visual analog scale (VAS), pain disability index (PDI), and percent global improvement. The primary outcome was VAS and PDI score changes from baseline at 6-month follow-up. All patients presented for their 6-month followup, with most having additional visits before and after. 
The VAS is an 11-point scale with 0 being no pain and 10 being worst possible pain. The PDI is comprised of 7 questions assessing the impact of chronic pain on activities such as recreation, self-care, and occupation. Scores range from 0 (no disability from pain) to 70 (worst disability from pain) (17). Percent global improvement scores ranged from $0 \%$ (no improvement) to $100 \%$ (maximum possible improvement).

\section{STATISTICAL ANALYSIS}

Descriptive statistics were used to evaluate variable frequencies. Within participant changes were evaluated using paired two-tailed Student's t-tests to calculate differences between baseline, 6-month, and greater than one-year VAS and PDI scores. Mean and standard deviations or percentages are reported for the full cohort. Threshold of significance was set at $\mathrm{P}<0.05$. A minimal clinically important difference

Table I. Demographic and Clinical Characteristics ( $N=$ 16 Patients). Values are mean and standard deviation or percentages. BMI: body mass index; PRP: platelet-rich plasma; MRI: magnetic resonance imaging.

\begin{tabular}{|c|c|}
\hline & $\mathrm{N}=16$ Patients \\
\hline Age & $65.1 \pm 9.6$ \\
\hline $\mathrm{BMI}$ & $28.5 \pm 6.1$ \\
\hline \multicolumn{2}{|l|}{ Laterality } \\
\hline Right & $11(68.7 \%)$ \\
\hline Left & $3(18.8 \%)$ \\
\hline Bilateral & $2(12.5 \%)$ \\
\hline \multicolumn{2}{|l|}{ Dominant Arm } \\
\hline Right & $16(100 \%)$ \\
\hline Symptom Duration (m) & $65.7 \pm 59.9$ \\
\hline Follow-up (m) & $12.1 \pm 5.6$ \\
\hline \multicolumn{2}{|l|}{ Sex } \\
\hline Male & $9(56 \%)$ \\
\hline Female & $7(44 \%)$ \\
\hline \multicolumn{2}{|l|}{ Previous Treatments } \\
\hline Physical Therapy & $16(100 \%)$ \\
\hline Steroid Injection & $15(94 \%)$ \\
\hline PRP Injection & $10(63 \%)$ \\
\hline \multicolumn{2}{|l|}{ Imaging } \\
\hline Plain Radiograph & $16(100 \%)$ \\
\hline MRI & $15(94 \%)$ \\
\hline
\end{tabular}

(MCID) value of 1.4-point change was used for VAS and 9.5-point change for PDI $(18,19)$. Descriptive values of proportion of participants meeting MCID are presented.

\section{RESULTS}

Demographics and clinical characteristics are depicted in table I. Most patients' (72\%) procedure was in the shoulder of the dominant arm and on average duration of symptoms was approaching 6 years. Follow-up was obtained in all participants for minimum of 6 months (average followup was $12.1 \pm 5.6$ months). Diagnostic imaging identified severe glenohumeral joint osteoarthritis and partial supraspinatus tears as the most prevalent pathologies, seen in $50 \%$ and $33 \%$ of shoulders, respectively (table II).

At baseline, mean VAS was $6.1 \pm 2.1$ and mean PDI was $21.4 \pm$ 9.2. There was significant improvement between baseline and 6-month follow up VAS $(\mathrm{p}<0.001)$ and PDI $(\mathrm{p}=0.02)$ (figures 1,2). For the 10 shoulders with greater than one-year follow-up, significant improvement in VAS $(\mathrm{p}<0.001)$ and PDI ( $\mathrm{p}=0.03)$ was observed. At 6 months, the MCID was met for 14 (78\%) of shoulders for VAS and $9(50 \%)$ of shoulders for PDI. At final follow-up, global improvement averaged 70\% (range 20-100\%). One minor complication was reported of transient contact dermatitis from the dressing applied to the harvest site. No other complications were observed or reported.

Table II. Pathology on Imaging ( $\mathrm{N}=18$ Shoulders).

\begin{tabular}{lll}
\hline \multicolumn{2}{l}{$\mathbf{N}=\mathbf{1 8}$ Shoulders } \\
\hline Glenohumeral Osteoarthritis & 4 & $22,20 \%$ \\
\hline Moderate & 9 & $50 \%$ \\
\hline Severe & & \\
\hline Rotator Cuff Pathology & & \\
\hline Supraspinatus & 2 & $11,10 \%$ \\
\hline Tendinosis & 6 & $33,30 \%$ \\
\hline Partial Tear & 2 & $11,10 \%$ \\
\hline Full tear & 5 & $27,80 \%$ \\
\hline Acromioclavicular Osteoarthritis & & \\
\hline Infraspinatus & 2 & $11,10 \%$ \\
\hline Tendinosis & 2 & $11,10 \%$ \\
\hline Partial Tear & & \\
\hline Subscapularis & 1 & $5,60 \%$ \\
\hline Partial Tear &
\end{tabular}




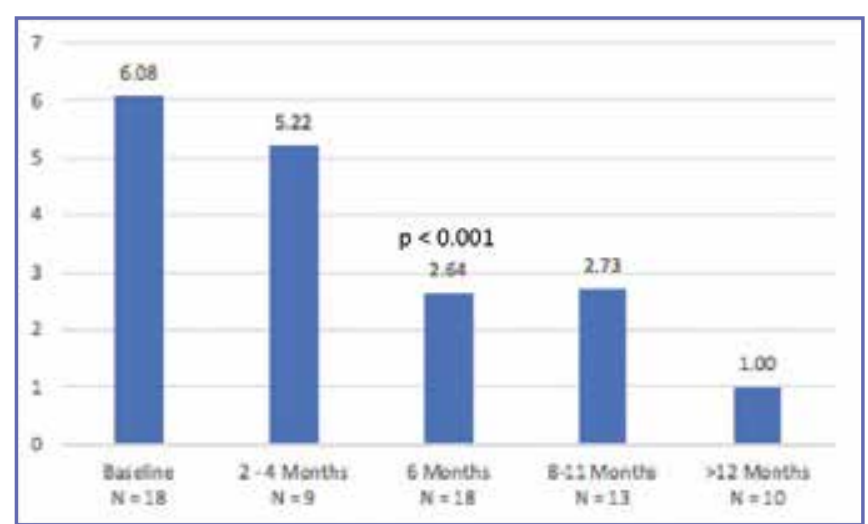

Figure 1. Visual Analog Scale Score Changes. Depicted are mean scores with corresponding number of patients for each follow-up time point.

\section{DISCUSSION}

Recalcitrant shoulder pain from osteoarthritis and rotator cuff pathology remains a common problem encountered in clinical practice. In this population of 15 patients, a majority reported significant improvements in pain and/or disability at six months using MCID criteria and no major adverse events were reported. These findings are notable as all patients had failed multiple prior conservative interventions, were not candidates or not ready for surgery, and reported pain and functional limitations for many months. The findings suggest MFAT may be successful in a portion of patients with shoulder pain from GHJ arthritis or RTC disease, with benefits measured 6 months from procedure. Current literature on MFAT use for shoulder pain remains limited. Striano et al. reported results on 18 patients with chronic shoulder pain treated with MFAT (20). Patients were followed for one year and significant improvement was noted for pain reduction and functional improvement using the Numeric Pain Scale and American Shoulder and Elbow Surgeons Score. Pain scores decreased from a baseline of 7.5 to 3.6 at one year. In the present study, we observed similar improvement in pain and disability. While statistical analyses could not be completed for after 6 months in our study, seven patients had follow-up times greater than 10 months with average point decreases of 4.2 and 4.6 in VAS and PDI, respectively, from baseline. A case report of a T10 complete paraplegic wheelchair user with chronic shoulder pain found improved pain and functional outcomes one-year post Lipogems ${ }^{\circledR}$ procedure (21). This allowed him to maintain independence and resume activities of daily living. Two studies report stromal vascular fraction (SVF) outcomes on shoulder pain via arthroscopy. Jo et al. treated 19 patients with varying doses of culture expanded SVF at the time

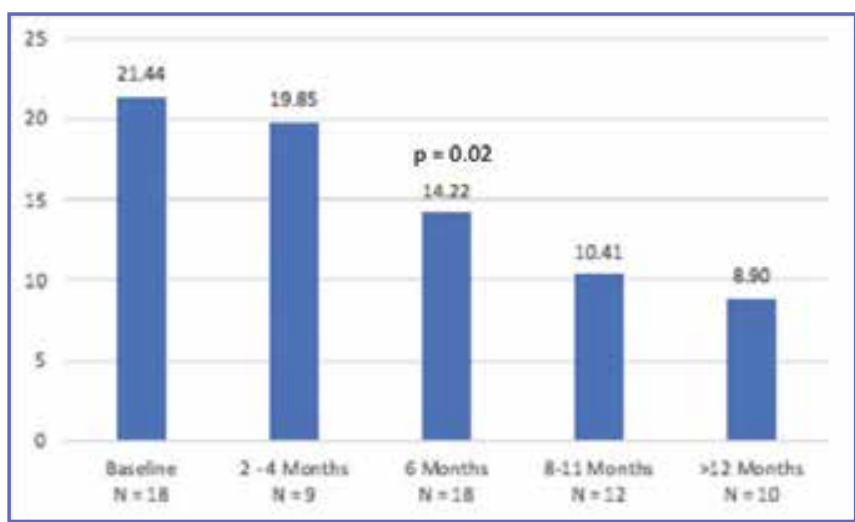

Figure 2. Pain Disability Index Score Changes. Depicted are mean scores with corresponding number of patients for each follow-up time point.

of an arthroscopic examination (22). Significant improvement was found at six months for the mid and high dose groups. The SVF used in this study was obtained through enzymatic digestion. Notably, culture-expanded cells are not approved for use in the United States as they violate the Federal Drug Administration's minimal manipulation regulations for MSCs. Kim et al. compared outcomes of surgical repair alone for rotator cuff tears against repair coupled with injection of adipose-derived MSCs loaded in fibrin glue. They found decreased re-tear rates in the surgery plus adipose-derived MSC group, but no significant differences in pain outcomes (23).

Multiple mechanisms have been proposed for how adiposederived signaling cells may improve pain and function for shoulder pathology. Immunomodulatory and anti-inflammatory properties secondary to a paracrine secretion of growth factors and cytokines likely contributes (11). In MFAT, these trophic properties can be attributed to the undifferentiated cells which are isolated from adult harvested adipose tissue (24). Additionally, tendon needling when treating rotator cuff tears invariably occurs and can have pain relieving properties (25).

While the findings are encouraging, results of this large case series should be interpreted with caution. Case series retrospective design is limited by sample size, low level of evidence (IV) and absence of a control group which precludes comparing outcomes amongst other treatment options. Selection bias of participants may influence results (including choice of procedure and out of pocket expense). MFAT is not a covered benefit under most commercial and government insurers in the United States, and out of pocket expense varies depending on which particular MFAT kit is used as well as overhead and demographics of the practice location. Often this amounts to a couple thousand US dollars. 
Notably no patients studied had received visco-supplementation with hyaluronic acid (HA) prior to their MFAT procedure. In vitro studies have demonstrated HA to potentially enhance tendon cell viability and proliferation (26). Additionally, in a review of 11 clinical studies, HA was found to improve pain and function in shoulders with rotator cuff tears (27). While often not covered by insurers in the United States, HA is typically a cheaper treatment option that can be considered prior to MFAT. The degree of osteoarthritis and rotator cuff tear severity may also influence results and was not controlled for in this study. Follow-up was obtained for a subset of patients to 6 months, and clinical results are often reported at time points 12 months after the procedure $(28,29)$. However, this report adds to the limited literature

\section{REFERENCES}

1. Luime JJ, Koes BW, Hendriksen IJM, et al. Prevalence and incidence of shoulder pain in the general population; a systematic review. Scandinavian Journal of Rheumatology 2004; 33 : 73-81.

2. Black EM, Higgins LD, Warner JJP. Value-based shoulder surgery: Practicing outcomes-driven, cost-conscious care. Journal of Shoulder and Elbow Surgery 2013; 22: 1000-9.

3. Meislin RJ, Sperling JW, Stitik TP. Persistent shoulder pain: epidemiology, pathophysiology, and diagnosis. American journal of orthopedics (Belle Mead, N.J.) 2005; 34: 5-9.

4. Oliva F, Piccirilli E, Bossa M, et al. I.S.Mu.L.T - Rotator cuff tears guidelines. Muscles Ligaments Tendons J 2015 Oct 1 [cited 2020 Feb 10];5(4):227-63. Available from: http://www. ncbi.nlm.nih.gov/pubmed/26958532.

5. Cadogan A, Laslett M, Hing WA, McNair PJ, Coates MH. A prospective study of shoulder pain in primary care: Prevalence of imaged pathology and response to guided diagnostic blocks. BMC Musculoskelet Disord 2011:12.

6. Dean BJF, Lostis E, Oakley T, Rombach I, Morrey ME, Carr AJ. The risks and benefits of glucocorticoid treatment for tendinopathy: A systematic review of the effects of local glucocorticoid on tendon. Vol. 43, Seminars in Arthritis and Rheumatism 2014: 570-6.

7. Petrillo S, Longo UG, Papalia R, Denaro V. Reverse shoulder arthroplasty for massive irreparable rotator cuff tears and cuff tear arthropathy: a systematic review. Musculoskelet Surg [Internet]. 2017 Aug [cited 2019 Sep 16];101(2):105-12. Available from: http://www.ncbi.nlm.nih.gov/pubmed/28444541.

8. Wilcox RB, Arslanian LE, Millett P. Rehabilitation following total shoulder arthroplasty. J Orthop Sports Phys Ther 2005 Dec [cited 2019 Sep 16];35(12):821-36. Available from: http://www.ncbi.nlm.nih.gov/pubmed/16848103.

9. Borg-Stein J, Osoria HL, Hayano T. Regenerative Sports Medicine: Past, Present, and Future (Adapted From the PASSOR Legacy Award Presentation; AAPMR; October 2016). PM R 2018 [cited 2019 Sep 7];10(10):1083-105. Available from: http://www.ncbi.nlm.nih.gov/pubmed/30031963.

10. DeChellis DM, Cortazzo MH. Regenerative medicine in the field of pain medicine: Prolotherapy, platelet-rich plasma ther- using Lipogems ${ }^{\circledR}$ and suggests further investigations using rigorous methodology (randomization, blinding, and larger sample sizes with more homogenous degree of pathology) would help understand the benefits of this procedure.

In conclusion, our results suggest MFAT may be helpful to improve pain and function in a subset of patients with chronic glenohumeral osteoarthritis and rotator cuff tears. No major complications were identified in our case series. Additional studies ideally prospective with a control group are needed for further evaluation of this treatment.

\section{CONFLICTS OF INTERESTS}

The author declare that they have no conflict of interests. apy, and stem cell therapy-Theory and evidence. Tech Reg Anesth Pain Manag 2011 Apr;15(2):74-80.

11. Freitag J, Bates D, Boyd R, Shah K, Barnard A, Huguenin $\mathrm{L}$, et al. Mesenchymal stem cell therapy in the treatment of osteoarthritis: Reparative pathways, safety and efficacy - A review, BMC Musculoskeletal Disorders BioMed Central Ltd 2016; 17.

12. Hudetz D, Borić I, Rod E, et al. Early results of intra-articular micro-fragmented lipoaspirate treatment in patients with late stages knee osteoarthritis: a prospective study. Croat Med J 2019 Jun 13;60(3):227-36.

13. Strioga M, Viswanathan S, Darinskas A, Slaby O, Michalek J. Same or not the same? comparison of adipose tissue-derived versus bone marrow-derived mesenchymal stem and stromal cells. Vol. 21, Stem Cells and Development 2012; 2724-52.

14. Bianchi F, Maioli M, Leonardi E, Olivi E, Pasquinelli G, Valente $\mathrm{S}$, et al. A new nonenzymatic method and device to obtain a fat tissue derivative highly enriched in pericyte-like elements by mild mechanical forces from human lipoaspirates. Cell Transplant 2013;22(11):2063-77.

15. Tremolada C, Colombo V, Ventura C. Adipose Tissue and Mesenchymal Stem Cells: State of the Art and Lipogems ${ }^{\circledR}$ Technology Development. Vol. 2, Current Stem Cell Reports. Springer International Publishing 2016; 304-12.

16. Padulo J, Oliva F, Frizziero A, Maffulli N. Muscles, ligaments and tendons journal - Basic principles and recommendations in clinical and field science research: 2016 update. Muscles Ligaments Tendons J 2016 Jan 1;6(1):1-5.

17. Jerome A, Gross RT. Pain disability index: construct and discriminant validity. Arch Phys Med Rehabil 1991 Oct [cited 2019 Sep 9];72(11):920-2. Available from: http://www.ncbi. nlm.nih.gov/pubmed/1929812.

18. Tashjian RZ, Deloach J, Porucznik CA, Powell AP. Minimal clinically important differences (MCID) and patient acceptable symptomatic state (PASS) for visual analog scales (VAS) measuring pain in patients treated for rotator cuff disease. J Shoulder Elb Surg 2009 Nov;18(6):927-32.

19. Soer R, Reneman MF, Vroomen PCAJ, Stegeman P, Coppes $\mathrm{MH}$. Responsiveness and minimal clinically important change 
of the pain disability index in patients with chronic back pain. Spine (Phila Pa 1976) 2012 Apr 15;37(8):711-5.

20. RD Striano GMNBKA. Refractory shoulder pain with osteoarthritis, and rotator cuff tear, treated with micro fragmented adipose tissue. Orthop Spine Sport Med 2018;2:014.

21. Cherian C, Malanga GA, Hogaboom N, Pollack MA, Dyson-Hudson TA. Autologous, micro-fragmented adipose tissue as a treatment for chronic shoulder pain in a wheelchair using individual with spinal cord injury: a case report. Spinal Cord Ser Cases 2019 Dec;5(1).

22. Jo CH, Chai JW, Jeong EC, Oh S, Kim PS, Yoon JY, et al. Intratendinous Injection of Autologous Adipose Tissue-Derived Mesenchymal Stem Cells for the Treatment of Rotator Cuff Disease: A First-In-Human Trial. Stem Cells 2018 Sep [cited 2019 Sep 9];36(9):1441-50. Available from: http://www. ncbi.nlm.nih.gov/pubmed/29790618.

23. Kim YS, Sung CH, Chung SH, Kwak SJ, Koh YG. Does an Injection of Adipose-Derived Mesenchymal Stem Cells Loaded in Fibrin Glue Influence Rotator Cuff Repair Outcomes? A Clinical and Magnetic Resonance Imaging Study. Am J Sports Med 2017 Jul 1;45(9):2010-8.

24. Bembo F, Eraud J, Philandrianos C, Bertrand B, Silvestre A, Veran J, et al. Combined use of platelet rich plasma \& microfat in sport and race horses with degenerative joint disease: Preliminary clinical study in eight horses. Muscles Ligaments Tendons J 2016 Apr 1;6(2):198-204.
25. Krey D, Borchers J, McCamey K. Tendon needling for treatment of tendinopathy: A systematic review. Phys Sportsmed 2015 Feb [cited 2019 Sep 9];43(1):80-6. Available from: http://www.ncbi.nlm.nih.gov/pubmed/25613418.

26. Gallorini M, Berardi AC, Berardocco M, et al. Hyaluronic acid increases tendon derived cell viability and proliferation in vitro: comparative study of two different hyaluronic acid preparations by molecular weight. Muscles Ligaments Tendons J [Internet]. 2017 [cited 2020 Feb 10];7(2):208-14. Available from: http://www.ncbi.nlm.nih.gov/pubmed/29264330.

27. Osti L, Buda M, del Buono A, Osti R, Massari L. Clinical evidence in the treatment of rotator cuff tears with hyaluronic acid. Vol. 5, Muscles, Ligaments and Tendons Journal. CIC Edizioni Internazionali s.r.l.; 2015;270-5.

28. Jo CH, Chai JW, Jeong EC, Oh S, Shin JS, Shim H, et al. Intra-articular Injection of Mesenchymal Stem Cells for the Treatment of Osteoarthritis of the Knee: A 2-Year Follow-up Study. Am J Sports Med [Internet]. 2017 Oct [cited 2019 Sep 16];45(12):2774-83. Available from: http://www.ncbi.nlm.nih. gov/pubmed/28746812.

29. Cattaneo G, De Caro A, Napoli F, Chiapale D, Trada P, Camera A. Micro-fragmented adipose tissue injection associated with arthroscopic procedures in patients with symptomatic knee osteoarthritis. BMC Musculoskelet Disord 2018 May 30 [cited 2019 Sep 16];19(1):176. Available from: http://www. ncbi.nlm.nih.gov/pubmed/29848328. 